\title{
Historias congeladas en el hielo polar
}

\author{
Guillermo N. Murray-Tortarolo
}

\section{Resumen}

Hielo...Raramente pensamos en él si no es para enfriar el agua de limón o el refresco. No obstante, cubre una décima parte de la superficie de nuestro planeta. Lejos de ser estático, el hielo guarda historias en las partículas de polvo y las burbujas que quedan atrapadas en su interior. En particular, la nieve de los casquetes polares y los glaciares ha retenido la historia de nuestra humanidad, de la vida, de la evolución de nuestro planeta y hasta del espacio.

En este artículo te contaré un par de las muchas, muchas historias que se encuentran congeladas en los hielos perpetuos terrestres. Primero, te llevaré a las partículas de plomo atrapadas en el Polo Norte y te hablaré de cómo reconstruyen la historia de nuestra humanidad. Después, daremos un salto espacial y te diré la manera en que el berilio congelado nos ayuda a entender la historia de nuestro Sol. Finalmente, conoceremos el riesgo que el cambio climático implica para este recurso histórico y la memoria que podríamos perder si el hielo polar se derrite.

Palabras clave: núcleo de hielo, testigo de hielo, isótopos, contaminación por plomo, glaciares, calentamiento global.

\section{FROZEN STORIES IN THE POLAR ICE}

\begin{abstract}
Ice... We rarely think about it, if it is not to cool our lemonade or sodas. However, it covers a tenth of the land area of Earth, and far from being still, ice keeps stories and memories in the dust particles and the air bubbles trapped inside it. In particular, ice sheets and glaciers have kept the story of humankind, life and even the evolution of Earth and our Solar System.

In this article I am going to tell you a couple of the many ,many stories that are frozen in perpetual terrestrial ice. First, I will talk about the lead particles trapped in the North Pole and how they can tell the history of humanity. Secondly, we will jump to space and reconstruct the story of our Solar System, through the frozen Beryllium records. Finally, I will tell you the risk that climate change possesses to this frozen memory, and all we could lose if ice sheets melt.
\end{abstract}

Keywords: ice core, isotopes, iron contamination, glaciers, global warming.

Dol: http://doi.org/10.22201/codeic.16076079e.2020.v21n2.a1 


\section{Guillermo N. Murray-Tortarolo}

Es Investigador Asociado "C" en el Instituto de Investigaciones en Ecosistemas y Sustentabilidad de la Universidad Nacional Autónoma de México (unam). Su tema principal de trabajo es la investigación ecológica a distintas escalas espacio-temporales. Le interesa entender los impactos del cambio y la variación climática sobre distintos procesos ecológicos y agronómicos de México y el mundo. También tiene un profundo interés y pasión por la divulgación de la ciencia y por la enseñanza de la misma.. 
Cuando pensamos en el hielo polar, muy posiblemente nos imaginemos a un oso blanco hibernando: metido en su cálida cueva, bajo tres o cuatro metros de nieve, en un enorme desierto blanco. Tal vez también recordemos a las focas que se sumergen en el fondo marino, por debajo de la capa congelada y algunos otros - tal vez la mayoría - piensen inmediatamente en Papá Noel y su casa de elfos. Pero lo que muy pocos se imaginan es que ese hielo milenario, que parece inerte y poco atractivo, es en realidad un gran historiador de nuestro planeta. Muchas de sus capas guardan la memoria de guerras, hambrunas, el surgimiento y caída de imperios completos, erupciones volcánicas y cambios globales en el clima. El registro del paso de nuestra humanidad se encuentra allí congelado, en la criósfera terrestre, esperando a ser excavado y estudiado. En este artículo te voy a platicar sobre este contador de historias congeladas y cómo el calentamiento global podría hacernos perder memorias aún desconocidas.

Una de las características más interesantes de los polos es que la precipitación es menor que en una zona desértica, pese a la enorme cantidad de nieve que aparentan tener. Los casquetes polares presentan Iluvias promedio de 350 milímetros al año; en comparación, el desierto de Chihuahua recibe entre 150 a 400 milímetros al año, pero con la diferencia de que cae en forma de nieve, la cual eventualmente se convierte en hielo. En otras palabras, el hielo que vemos en el Polo Norte o Sur se ha acumulado allí a través de miles de millones de años.

Al caer, la nieve hace dos cosas: atrapa polvo de la atmósfera y encierra moléculas de aire dentro de su estructura. Lo interesante es que, debido a diferencias en la densidad del hielo y la nieve, así como la falta de actividad biológica, las capas de nieve nuevas no se mezclan con las del pasado, que pueden ser del año anterior o, incluso, las de hace un millón de años. Como resultado, si excavamos unos metros de este hielo tendremos una radiografía del estado de la atmósfera de hace un par de milenios y si intentamos algunos kilómetros, jtendremos la historia atmosférica de cientos de miles de años!

Además, esta memoria congelada captura toda clase de partículas, por ejemplo: dióxido de carbono, metano, plomo y oxígeno. Cada elemento, isótopo y molécula viene con su propia historia, atrapada por millones de años, y de la que es necesario descifrar lo que nos quieren contar. Es como si tuvieran su propia contraseña que necesita ser descifrada para acceder a una memoria y una historia milenaria. Este es justamente el trabajo de algunos científicos (glaciólogos, geólogos, geofísicos y biólogos): entender y descifrar el lenguaje, asî como la historia que nos cuenta cada partícula en el hielo.

Se ha avanzado muchísimo en este campo; por ejemplo, ahora se sabe que el oxígeno dice cuánto llovía en antaño o que el dióxido de carbono y metano indican qué tan caliente era nuestro planeta. Sin embargo, todavía queda mucho por descifrar y cada día salen a la luz nuevos relatos de nuestro pasado congelado. 
Hoy les contaré dos de los relatos más recientes y fascinantes que los científicos lograron descifrar este año: el del plomo y cómo se liga profundamente con las actividades mineras de la humanidad; y el del berilio y su relación con la actividad solar.

\section{Plomo congelado y la minería}

Durante la historia de nuestro planeta, el plomo ha estado prácticamente enterrado. De hecho, fuera de las por poco invisibles partículas enviadas por las erupciones volcánicas, este elemento era casi inexistente en la atmósfera antes del origen de la industria humana. Una vez que la humanidad comenzó a desarrollarse, aumentó la fascinación por los metales preciosos, las joyas, y después el petróleo y el carbón, todos ellos ubicados en el subsuelo. Para satisfacer esta creciente necesidad, comenzamos a darle vuelta a la tierra (y a la Tierra), a hacer hoyos, huecos, túneles para sacar todo lo que estaba debajo.

Aún no existía la maquinaria, por lo que se emplearon esclavos para picar piedra en búsqueda de minerales preciosos. Se originó una economía mundial basada en el oro y con ella prosperaron y cayeron imperios, y, por otro lado, el plomo se usó para crear armamentos y construcciones. De cada pedacito de tierra obtenido se desprendió polvo; diminutas partículas se fueron volando por todos lados y algunas de ellas incluso llegaron a los polos y a los hielos perpetuos de los glaciares alpinos. Con esto volvemos a nuestra historia congelada y al hielo perpetuo, que se dedicó a recolectar ese polvo año con año, registrando la historia de nuestra actividad minera y de nuestro frenesí por lo enterrado.

Allí el plomo se mantuvo congelado dentro del hielo glaciar y polar, por miles de años, hasta que el pionero Joseph R. McConell y colaboradores decidieron sacarlo para descifrar su historia. Ellos colectaron núcleos de hielo en cinco puntos de Groenlandia y en hielos perpetuos rusos (ver imagen 1), donde midieron la concentración de plomo de cada centímetro y, mediante otros indicadores, determinaron el año aproximado de proveniencia. Es decir, para cada uno de los últimos 2100 años, realizaron un estimado de la cantidad de plomo que circulaba en la atmósfera. Ligaron cada período de cambio en las concentraciones de plomo con lo que sucedía en la historia de la humanidad, particularmente en Europa. Sus resultados fueron publicados en la revista Proceedings of the National Academy of Sciences (PNAS) en marzo de $2019 \mathrm{y}$ rápidamente hicieron eco en las redes sociales. 
Imagen 1. Cortesía de la NASA http://earthobservatory.nasa. gov/Features/Paleoclimatology IceCores/
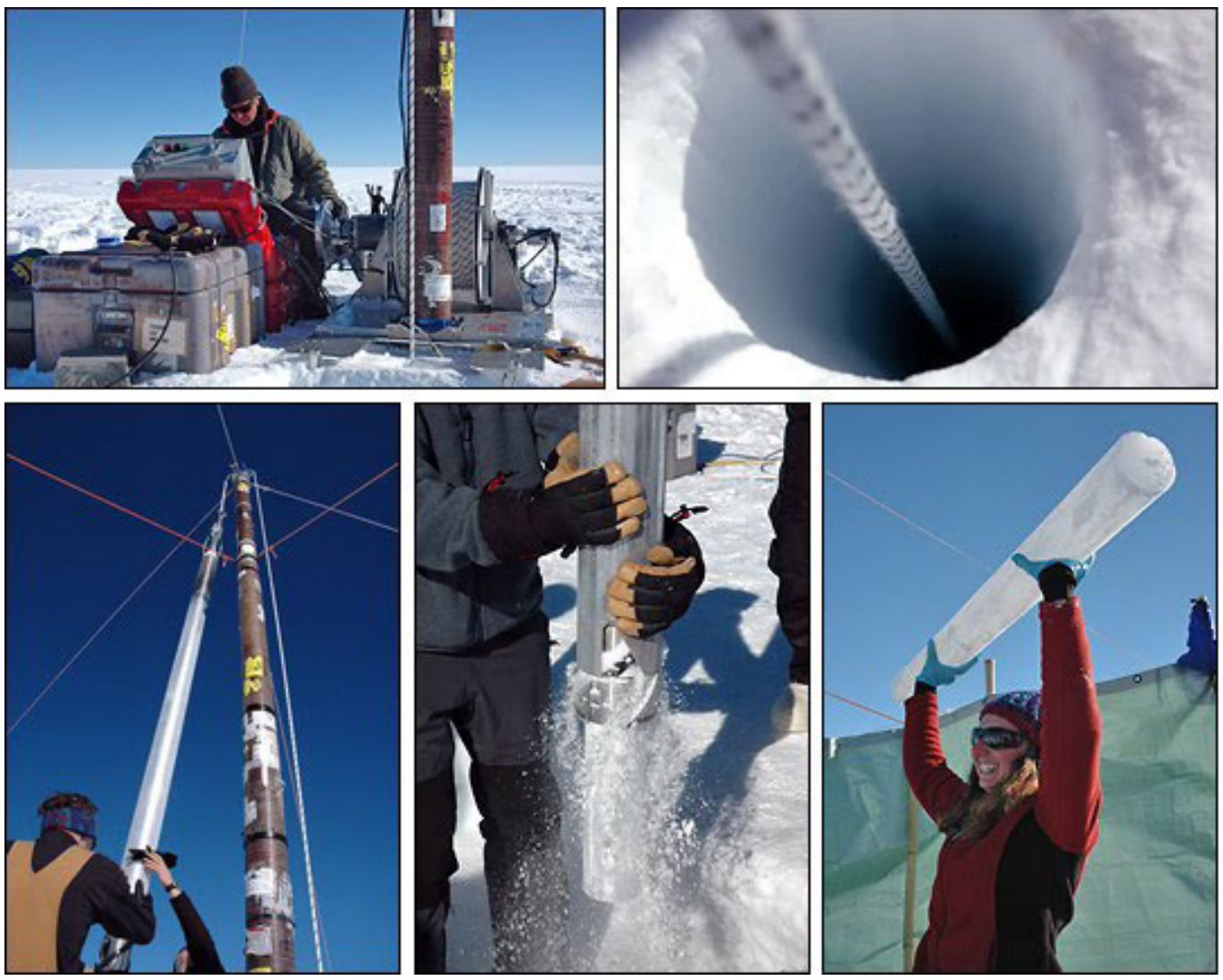

Los autores mostraron cómo diferentes momentos de la humanidad modificaron la actividad minera en Europa y Asia. Por ejemplo, durante el surgimiento y auge del Imperio romano la concentración de plomo congelado aumentó casi diez veces como resultado de la creación de armamento, decayó con cada una de las plagas que azotó al imperio, resurgió en períodos de crecimiento económico y finalmente llegó a un mínimo con su caída. De allí permaneció estable hasta el reinado de Carlomagno y la Edad Media, donde creció exponencialmente por casi mil años (cien veces en total), consecuencia del gusto europeo por la plata, hasta la llegada de la peste bubónica y las hambrunas de la tardía Edad Media que trajeron una caída abrupta en la actividad humana y, por lo tanto, en la concentración de plomo polar. Finalmente, con el inicio y auge de la Revolución Industrial, se dio el último gran brinco pues la concentración registrada de plomo incrementó 228 veces como resultado de la extracción de carbón y petróleo. En los últimos tiempos, la concentración atmosférica nuevamente se disparó (por el uso de gasolinas con plomo durante la década de los ochenta), para caer abruptamente al inicio de 1990, cuando se prohibió este metal en los combustibles y se sustituyó por productos de nitrógeno. Actualmente, el depósito de plomo se ha estabilizado, pero sigue siendo 60\% mayor que antes de la Edad Media. 
La belleza de esta simple línea de tiempo recae en pensar justamente todo lo que hay detrás. La marcada tendencia de aumento temporal coincide con el surgimiento de nuevas tecnologías basadas en la industria extractiva, del hallazgo de nuevas minas, la importación de minerales de América y períodos de riqueza económica. En particular, destaca la creación de una economía basada en monedas y el auge de los mercados de plata. No obstante, todos los momentos de incremento parecen culminar en grandes guerras, enfermedades o hambrunas, que cambian la tendencia. Sobre todo, la peste bubónica, que acabó con la tercera parte de la población europea, tiene una señal clara en el registro, a causa de una caída en las actividades mineras de la región.

Esta bella historia es el hallazgo más reciente de los cuentos del hielo, aunque hace unos años, científicos de distintas partes del mundo hallaron otro elemento que, lejos de contarnos lo que pasa aquí en la Tierra, nos narra la historia de nuestro sistema solar, especialmente del Sol. Veamos de qué se trata.

\section{El berilio fugaz y los rayos cósmicos}

Casi todos los rayos cósmicos que recibe la Tierra provienen del exterior del sistema solar, causados principalmente por la explosión de supernovas. El hielo, como el gran historiador que es, procura registrar su paso. Esto sucede ya que al llegar a la atmósfera terrestre, los rayos interactúan con elementos que se encuentran allí (principalmente nitrógeno) y generan la creación de algo muy particular: isótopos de berilio diez (10Be), causados exclusivamente por esta interacción y aunque se pueden producir en las reacciones nucleares de nuestros reactores y bombas, naturalmente no se encuentran de otra forma en la Tierra. Algo curioso del berilio es que es un elemento que prácticamente no interactúa con nada ni con nadie. No es usado por la vida, no tiene injerencia en reacciones químicas y tampoco desaparece. Como resultado, así como se forma en la atmósfera, así se diluye en el agua y, como habrás adivinado, una parte de éste termina depositado en el hielo polar (ver imagen 2).

Lo notable es que la cantidad de radiación cósmica recibida por la Tierra no es siempre la misma. Las variaciones en el campo magnético solar llevan a fluctuaciones en la cantidad de rayos cósmicos que entra a nuestro planeta. Entre más débil sea este "escudo protector", mayor será la cantidad de rayos que recibiremos. De esta forma, períodos con baja actividad solar se traducen en un aumento de berilio en el hielo y viceversa.

Por lo tanto, si midiéramos la concentración del isótopo de berilio en un núcleo de hielo, se podría reconstruir la historia de la actividad solar pasada. No obstante, este isótopo es casi inexistente, con cerca de diez mil átomos en un gramo de hielo (que tiene $9 \times 10^{22}$ átomos, o, en otras palabras, menos del $0.0000000000001 \%$ de berilio en cada gramo de hielo) y hasta hace muy poco no teníamos la tecnología para poder medirlo. 
Friedhelm Steinhilber y colaboradores fueron los primeros en tener acceso a la tecnología necesaria. Ellos diseñaron el experimento para colectar, medir y reportar las concentraciones de dicho isótopo en el hielo polar para entender la historia de nuestro Sol. Sus resultados, publicados en la revista pNAs en abril de 2012, representan la primera reconstrucción de los cambios en la irradiancia solar en los últimos 9,000 años. Los autores mostraron ciclos en los picos de actividad solar cada mil años, los cuales han disminuido lentamente para llegar a un mínimo en la actualidad. Los resultados arrojaron que el calentamiento global en que vivimos hoy en día no es consecuencia de una actividad solar extraordinaria, pues ésta se encuentra en su valor más bajo de los últimos 9,000 años.

Imagen 2. Proceso de formación del berilio 10 y su acumulación en el hielo polar. Recuperada de: https://doi. org/10.1073/pnas.1118965109

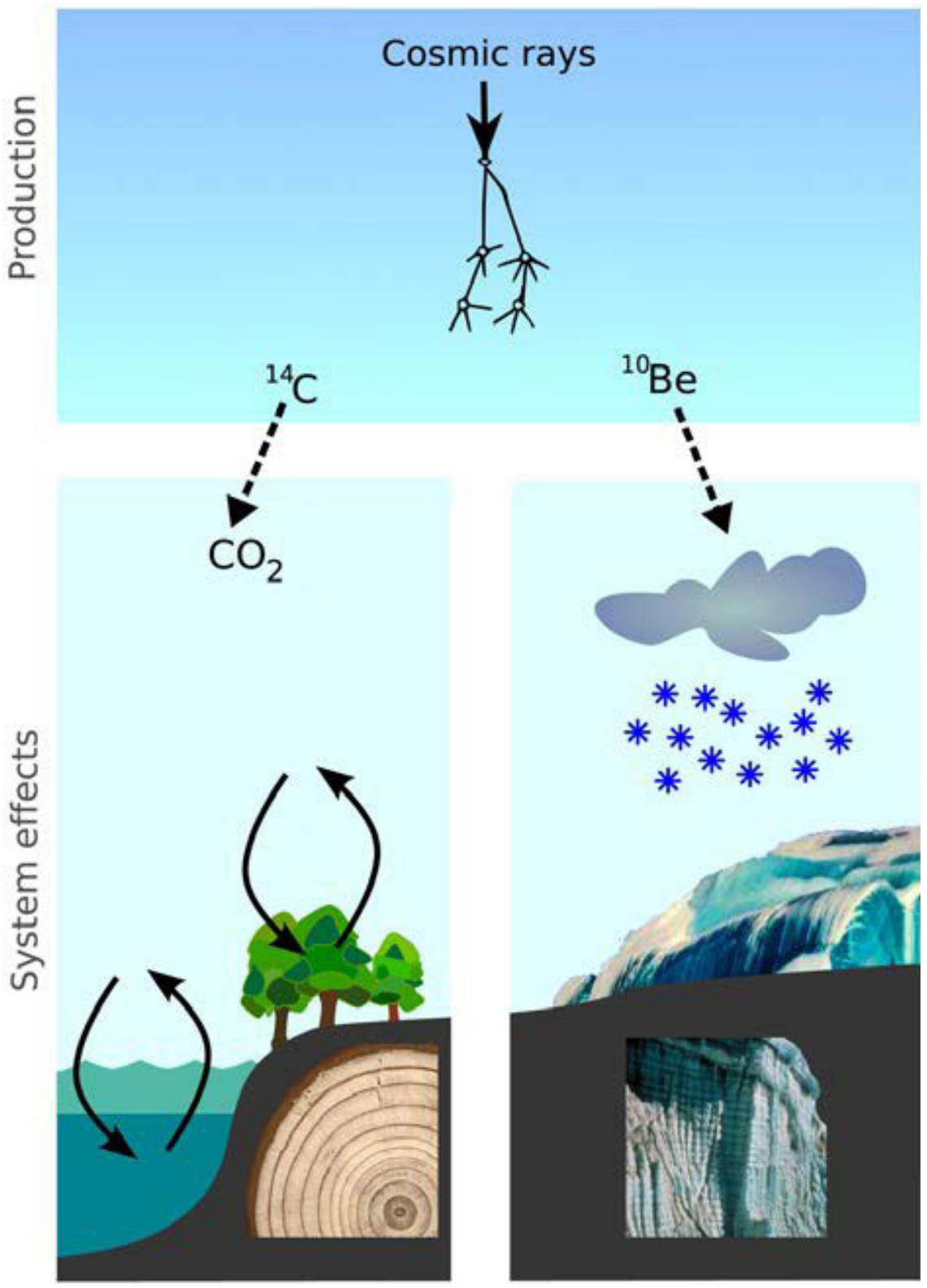




\section{Una memoria que se derrite}

Ninguna memoria es eterna, especialmente cuando decidimos guardarla en el hielo de un planeta que aumenta de temperatura día con día. El impacto del calentamiento global al que nos enfrentamos no sólo incluye el derretimiento de los casquetes polares (y la consecuente expansión del océano); sino también la pérdida de una memoria histórica guardada en sus burbujas y polvo. Se trata de un recurso fundamental para el equilibrio térmico y radiativo de la tierra, así como para el recuento de la historia de la vida terrestre y de nuestra humanidad, que se pierde lentamente. Desconocemos cuántas aventuras estarán guardadas en otros elementos y qué otras cosas aún nos faltan por descubrir allí atrapadas y congeladas. Por suerte, algunos centros de investigación como el Scott Polar Research Institute en el Reino Unido o el National Ice Core Laboratory en Estados Unidos, preservan núcleos para futuras investigaciones. Al menos así, parte de la memoria histórica polar se encuentra resguardada para descubrirla en el futuro.

Como última nota, si consideran el hielo polar como lejano, deben saber que no se encuentra únicamente en lugares remotos, los glaciares que están en nuestras propias montañas también resguardan muchas anécdotas: los del Popocatépetl e Iztaccíhuatl (ver imagen 3), Pico de Orizaba, Nevado de Toluca y Nevado de Colima podrían contener la historia del paso de todas las civilizaciones de Mesoamérica o del clima regional y sus cambios en los últimos milenios. Éstos se están perdiendo a un ritmo demasiado rápido y con ello las historias que contienen; por ejemplo, se estima que el Iztaccíhuatl es el de mayor riesgo de perder todo el hielo que lo cubre en los próximos cinco o diez años, y que aun los más altos, como el Pico de Orizaba, se extingan en este siglo.

Imagen 3. Glaciares del Popocatépetl e Iztaccíhuatl. Recuperada de: https://www. gaceta.unam. $\mathrm{mx} / \mathrm{glaciares}$ mexicanos-a-punto-deextinguirse/

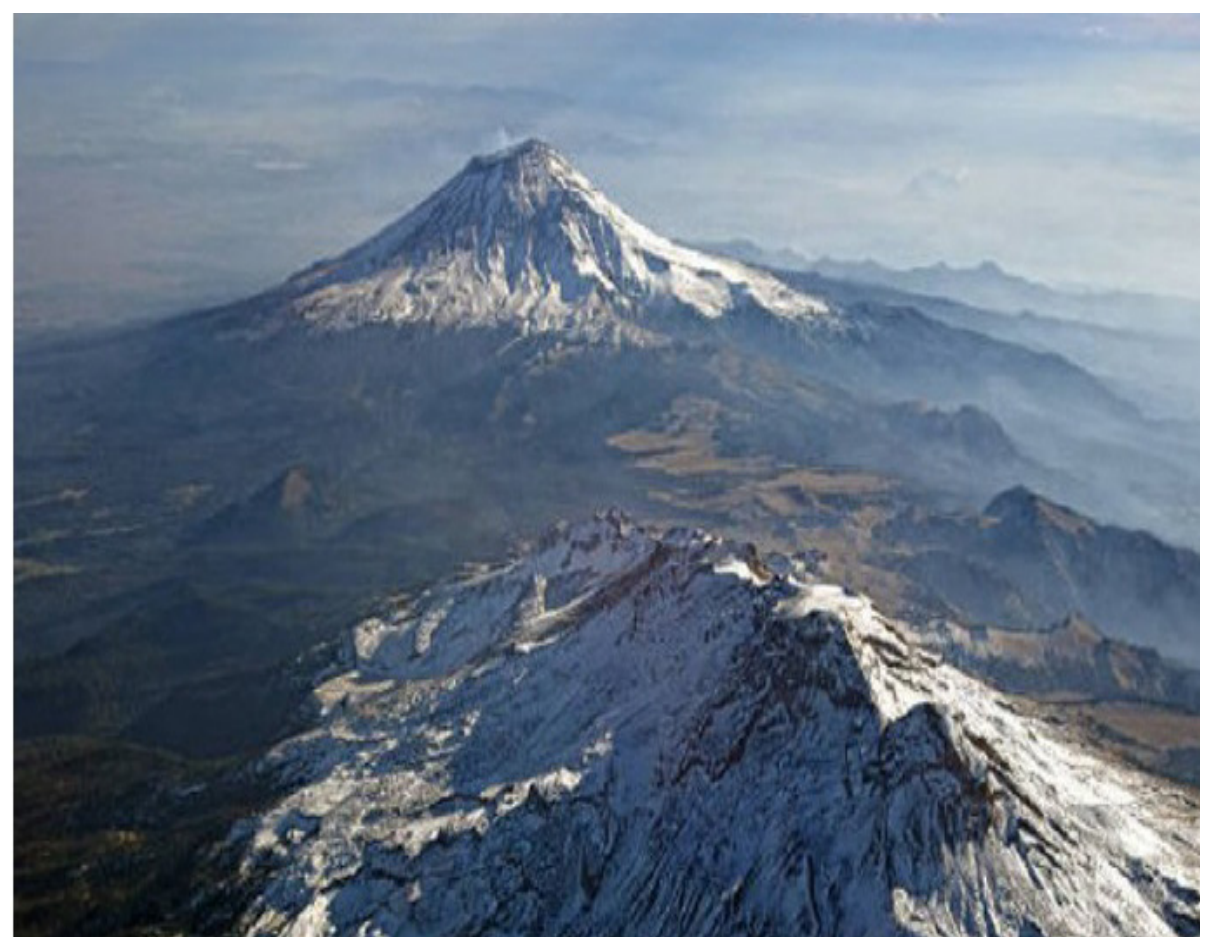


A pesar de que existe el riesgo de perder estas memorias, también hay una oportunidad de hacer algo por ellas. Hacen falta nuevos científicos con ganas de subir miles de metros a extraer hielo para llevar las muestras congeladas a un laboratorio y a descifrar los cuentos de este helado historiador. Y quién sabe, a lo mejor podrías ser tú el próximo glaciólogo que descifre una nueva historia.

\section{Referencias}

* McConnell, J. R., Wilson, A. I., Stohl, A., Arienzo, M. M., Chellman, N. J., Eckhardt, S., Thompson, E. M., Pollard, A. M. \& Steffensen, J. P. (2018). Lead pollution recorded in Greenland ice indicates European emissions tracked plagues, wars, and imperial expansion during antiquity. Proceedings of the National Academy of Sciences, 115(22), 5726-5731. Dol: https://doi.org/10.1073/pnas.1721818115.

* Petit, J. R., Jouzel, J., Raynaud, D., Barkov, N. I., Barnola, J. M., Basile, I., Bender, M, Chappellaz, J., Davis, M., Delaygue, G., Delmotte, M., Kotlyakov, V. M., Legrand, M., Lipenkov, V. Y., Lorius, C., PÉpin, L., Ritz, C., Saltzman, E. \& Stievenard, M. (1999). Climate and atmospheric history of the past 420,000 years from the Vostok ice core, Antarctica. Nature, 399(6735), 429. Dol: https://doi.org/10.1038/20859.

- Steinhilber, F., Abreu, J. A., Beer, J., Brunner, I., Christl, M., Fischer, H., Heikkilä, U., Kubik, P. W., Mann, M., McCracken, K. G., Miller, H., Miyahara, H., Oerter, H. \& Frank Wilhelms (2012). 9,400 years of cosmic radiation and solar activity from ice cores and tree rings. Proceedings of the National Academy of Sciences, 109(16), 5967-5971. Dol: https://doi.org/10.1073/pnas.1118965109.

\section{Cómo citar este artículo}

* Murray-Tortarolo, Guillermo N. (2020). Historias congeladas en el hielo polar. Revista Digital Universitaria (RDU). Vol. 21, núm. 1 marzo-abril. Dol: http://doi.org/10.22201/ codeic.16076079e.2020.v21n2.a1.

Recepción: 01/08/2019. Aprobación: 22/11/2019 